\title{
Absolute retinal blood flowmeter using a laser Doppler velocimeter combined with adaptive optics
}

\author{
Frederic Truffer, ${ }^{a}$ Martial Geiser $\odot,{ }^{a}, *$ Marc-Antoine Chappelet, ${ }^{b}$ \\ Helene Strese, ${ }^{\mathrm{a}}$ Gilbert Maître $\odot,{ }^{\mathrm{a}}$ Serge Amoos, ${ }^{\mathrm{a}}$ Florent Aptel, ${ }^{\mathrm{b}, \mathrm{c}, \mathrm{d}}$ and \\ Christophe Chiquet $\oplus^{\text {b,c,d }}$ \\ ${ }^{a}$ University of Applied Sciences and Arts of Western Switzerland, \\ Institute of System Engineering, Sion, Switzerland \\ ${ }^{b}$ University Hospital, Department of Ophthalmology, Grenoble, France \\ ${ }^{\mathrm{c}}$ Grenoble Alpes University, Faculty of Humanities, Health, Sport and Society, Grenoble, France \\ ${ }^{\mathrm{d}}$ Inserm, CHU Grenoble Alpes, HP2, Grenoble, France
}

\begin{abstract}
Significance: The development of a technique allowing for non-invasive measurement of retinal blood flow (RBF) in humans is needed to understand many retinal vascular diseases (pathophysiology) and evaluate treatment with potential improvement of blood flow.
\end{abstract}

Aim: We developed and validated an absolute laser Doppler velocimeter (LDV) based on an adaptive optical fundus camera that provides simultaneously high-definition images of the fundus vessels and absolute maximal red blood cells (RBCs) velocity to calculate the absolute RBF.

Approach: This new absolute LDV is combined with the adaptive optics (AO) fundus camera (rtx1, Imagine Eyes ${ }^{\circledR}$, Orsay, France) outside its optical wavefront correction path. A 4-s recording includes 40 images, each synchronized with two Doppler shift power spectra. Image analysis provides a vessel diameter close to the probing beam, and the velocity of the RBCs in the vessels are extracted from the Doppler spectral analysis. A combination of these values gives an average of the absolute RBF.

Results: An in vitro experiment consisting of latex microspheres flowing in water through a glass capillary to simulate a blood vessel and in vivo measurements on six healthy humans was done to assess the device. In the in vitro experiment, the calculated flow varied between 1.75 and $25.9 \mu \mathrm{L} / \min$ and was highly correlated $\left(r^{2}=0.995\right)$ with the flow imposed by a syringe pump. In the in vivo experiment, the error between the flow in the parent vessel and the sum of the flow in the daughter vessels was between $-11 \%$ and $36 \%$ (mean \pm sd, $5.7 \pm 18.5 \%$ ). RBF in the main temporal retinal veins of healthy subjects varied between 0.9 and $13.2 \mu \mathrm{L} / \mathrm{min}$.

Conclusions: The AO LDV prototype allows for the real-time measurement of absolute RBF derived from the retinal vessel diameter and the maximum RBCs velocity in that vessel.

(C) The Authors. Published by SPIE under a Creative Commons Attribution 4.0 Unported License. Distribution or reproduction of this work in whole or in part requires full attribution of the original publication, including its DOI. [DOI: 10.1117/1.JBO.25.11.115002]

Keywords: laser Doppler velocimetry; retinal blood flow; absolute retinal blood flow; fundus camera.

Paper 200254R received Aug. 9, 2020; accepted for publication Nov. 4, 2020; published online Nov. 24, 2020.

\section{Introduction}

The inner retina is supplied by the retinal circulation, which is characterized by lower flow than the choroid, high-oxygen extraction, absence of any anastomosis, and autonomic innervation. The retinal blood flow (RBF) adaptation to the perfusion pressure is modulated by a fine

*Address all correspondence to Martial Geiser, martial.geiser@hes-so.ch 
autoregulation based on a balance between myogenic (via endothelium) and metabolic (via neurons and glia) mechanisms. ${ }^{1}$ Abnormal variations of RBF have been reported in a variety of ocular diseases including age-related macular degeneration ${ }^{2-4}$ and glaucoma,${ }^{5}$ but also in systemic diseases such as diabetes ${ }^{6,7}$ and systemic hypertension. ${ }^{8-10}$ Comprehensive knowledge of RBF, essential for understanding the pathophysiology of ocular and systemic diseases, is also the key to the evaluation of therapeutic strategies. ${ }^{11}$

RBF depends on the vascular cross section and the velocity of red blood cells (RBC). Several techniques have been developed to estimate or to measure RBF in the human eye such as fluorescein angiography, ${ }^{12}$ laser Doppler velocimeter (LDV),${ }^{13-17}$ Doppler optical coherence tomography, ${ }^{18-20}$ optical coherence angiography ${ }^{21,22}$ (OCT-A), and laser Doppler holography ${ }^{23}$ (LDH). Although OCT-A is able to show where blood is circulating, it does not estimate velocity of RBC and thus gives no information about the flow rate. ${ }^{24} \mathrm{LDH}$ has the potential to evaluate the velocity within the vessel but mainly depends on the frame rate, which must be in the order of $100 \mathrm{kHz}$. Although the canon laser blood flowmeter ${ }^{17}$ systems allow for quantification of blood flow using one acquisition, the LDV systems developed so far allow for the independent acquisition of blood velocity and retinal vascular diameters. ${ }^{14-16,25,26}$ Simultaneous measurements of retinal vascular diameters and velocity are highly advisable due to physiological variability of these parameters.

The measurement of blood vessel diameter is based on fundus images combined with image analysis using densitometry. ${ }^{27,28}$ The recent and innovative technology using adaptive optics (AO) aims at correcting low-order and high-order ocular aberrations, enhances performance of the optical systems, and allows for high-resolution imaging of retinal vessels. For instance, the $\mathrm{rtx} 1$ instrument provides in vivo retinal images with high lateral resolution (1.6 $\mu \mathrm{m}$ per pixel) and a quantitative analysis of microvascular structures, especially the measurement of retinal arteriolar wall thickness. ${ }^{29}$

To overcome the limitations of the present technologies, we developed an approach based on simultaneous measurements of retinal vessel diameters using AO technology that provides 16-bit images $^{29}$ and is able to measure the inner retinal vessel and blood velocity using bidirectional LDV. In this paper, we report in vitro and in vivo experiments investigating the validity of blood flow rate measured by this new prototype (aoLDV).

\section{Materials and Methods}

\subsection{Description of the Instrument}

\subsubsection{Camera with adaptive optics}

Images were obtained using the commercially available AO retinal camera, ${ }^{30,31}$ which measures and corrects wavefront aberrations with a 750-nm super luminescent diode source and an AO system operating in a closed loop. A $4^{\circ} \times 4^{\circ}$ fundus area (i.e., $\sim 1.2 \times 1.2 \mathrm{~mm}^{2}$ in emmetropic eyes) is illuminated at $840 \mathrm{~nm}$ by a light emitting diode with low temporal coherence, and a stack of 40 fundus images is acquired in $4 \mathrm{~s}$ (10 images per second) by a charge-coupled device camera. The rtx 1 provides a continuous fundus image that is used to align the probing beam. Its intensity is $100 \mu \mathrm{W}$ at the cornea, well below the maximum permissible exposure of $700 \mu \mathrm{W}$ at that wavelength (ANSI Z136.1-2000).

\subsubsection{Principle of bidirectional laser Doppler velocimetry}

The principle of absolute LDV for the human eye was first published by Riva et al. ${ }^{14}$ One probing beam with direction $\vec{k}_{i}$ is focused on a blood vessel, in which laminar flow is assumed [Fig. 1(a)]. Light is backscattered and Doppler frequency shifted due to the movement of RBCs within the vessel. Two scattered beams, $A$ and $B$, are selected with a dedicated pupil so that the angle $\alpha$ between both beams is defined by the optical system and the length of the eye. The vector of the principal ray of each beam and the velocity of the blood in the vessel are all in the same plane. Assuming a quadratic velocity profile ${ }^{32}$ of the RBCs moving in the vessel, the power spectrum of 

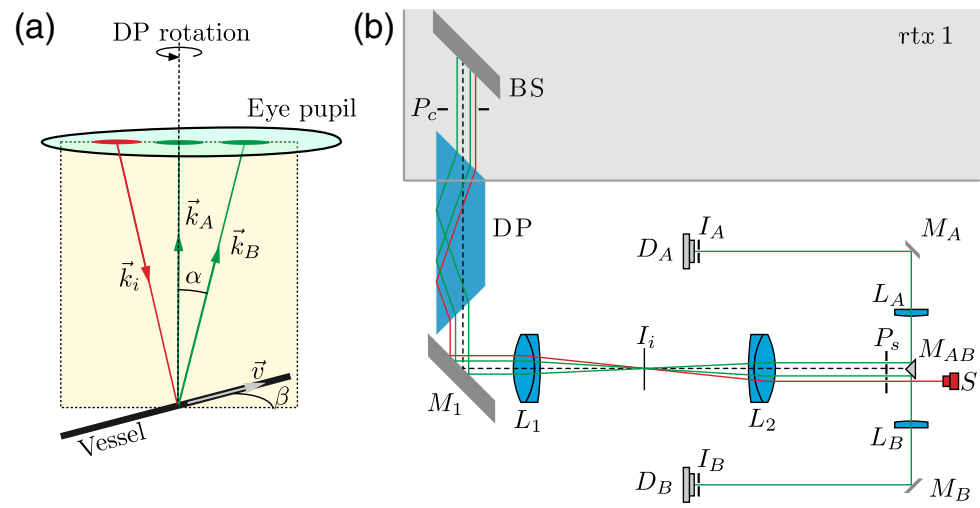

Fig. 1 (a) Principle of absolute LDV: $\vec{k}_{i}$ represents the probing beam direction and $\vec{k}_{A}$ and $\vec{k}_{B}$ are the scattered directions that are selected within the pupil. The three vectors $\vec{k}_{i}, \vec{k}_{A}$, and $\vec{k}_{B}$ are on the same plane that makes an angle $\beta$ with $\vec{v}$. (b) Optical system: except the BS (830 nm RazorEdge Dichroic laser-flat BS, Semrock, USA), which is part of the rtx1, all optical elements are mounted on a separate system that is attached to the rtx 1 and aligned with respect to it. $P_{x}$ are the pupil planes, $I_{x}$ are image planes, $M_{x}$ are mirrors, $L_{x}$ are lenses, and $D_{x}$ are detectors. The DOE (on a slider not seen in the image) is placed at $P_{s}$.
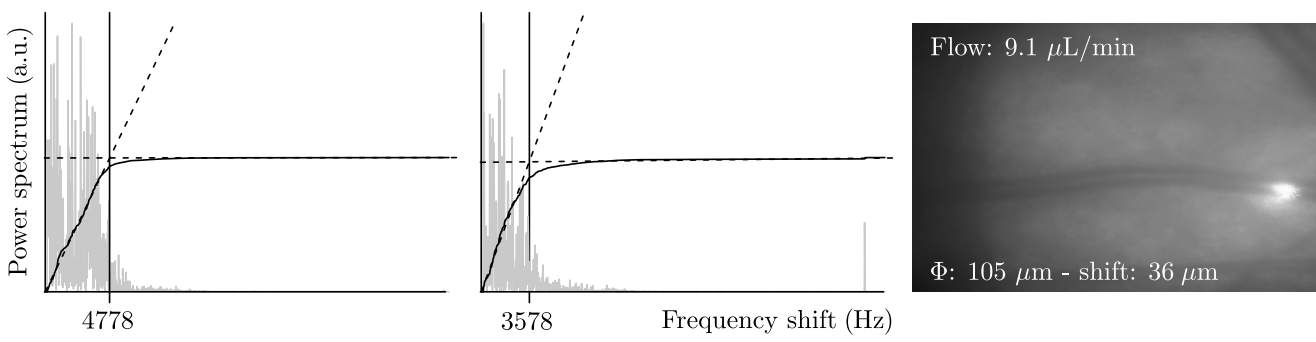

Fig. 2 Single-measurement example. Graphs: graphical explanation of the procedure used to find the maximum frequency shift of both signals. The function $r_{c}[i(f)]$ (solid black line) is derived from the power spectrum (gray line). Dashed lines are fitted lines of $r_{c}[i(f)]$ at the beginning and at the end of the frequency domain. The crossing of both fitted lines defines the maximum frequency shift. Image: corresponding fundus image with the calculated value of the flow and the diameter.

the electronic signal has a theoretical step shape with a sharp edge at the maximum frequency shift (see spectra of Fig. 2). The maximum frequency shift corresponds to the maximum velocity of the blood $v_{\max }$ in the center of the vessel. In this case, it can be shown that

$$
v_{\max }=\lambda \cdot \frac{\left|f_{A}-f_{B}\right|}{n \cdot \alpha \cdot \cos \beta},
$$

where $f_{A}$ and $f_{B}$ are the maximum frequency shifts of beams $A$ and $B$, respectively, $\lambda$ is the wavelength of the laser source, and $n$ is the refractive index of the plasma surrounding the RBCs. The angle $\alpha$, between the two scattered beams, is calculated using the formula $\alpha=\arctan (x / L)$, where $x$ represents the distance between the beam position ( $A$ and $B$, Fig. 1$)$ at the eye pupil and $L$ is the axial eye length assumed to be $23.95 \mathrm{~mm}^{33}$ and is set identical for all subjects. By observing the line of the probing beam on the fundus image acquired by the rtx 1 camera, the angle $\beta$ [Fig. 1(a)] is adjusted to zero by rotating a Dove prism (DP) [Fig. 1(b)] so that it has no influence on the calculation of $v_{\max }$. In practice, an angle $\beta$ up to $10 \mathrm{deg}$ (inducing an error of $1.5 \%$ ) is acceptable to obtain a reliable measurement of $v_{\max }$.

\subsubsection{Optical layout of the velocimeter}

The LDV optical system is introduced inside the illumination path with the beam splitter (BS) [Fig. 1(b)] and is located outside the adaptive optical path of the rtx1. A 830-nm laser source $S$ 
focused at infinity enters the optical system close to the edge of the pupil of the instrument, passes through the LDV and rtx1 optical systems to enter the pupil of the subject, and focuses on a vessel. The probing beam diameter on the retina is about $50 \mu \mathrm{m}$. Part of this light is backscattered by the vessel and the RBCs and goes back through the pupil of the eye to the same optical system up to the image of the pupil plane $P_{s}$ where two holes select the two specific

directions $\vec{k}_{A}$ and $\vec{k}_{B}$. A small reflecting prism $M_{A B}$ deflects each beam to lenses $L_{A}$ and $L_{B}$, which focus each beam on a pinhole at the image plane $I_{A}$ and $I_{B}$ corresponding to about $100 \mu \mathrm{m}$ at the retinal plane. Detectors $D_{A}$ and $D_{B}$ (APD C5460, Hamamatsu) are placed just behind the pinholes. A diffractive element (DOE) placed at $P_{s}$ converts the spot in the fundus into a line. Then the (DP) is rotated to visually align this line with the vessel on the screen of the rtx 1 (minimizing $\beta$ ). Lenses $L_{1}$ and $L_{2}$ form a telescope that relays the $\mathrm{rtx} 1$ pupil $P_{c}$ to the pupils $P_{s}$ of the LDV optical system with a unity magnification.

\subsubsection{Signal acquisition and electronics}

The electrical signals from the two detectors are connected to an acquisition card (USB-6356, National Instruments) and are sampled at $120 \mathrm{kHz}$ and Fourier analyzed. The direct current corresponding to the light intensity of a collected scattered light beam was removed by an analog filtering before the acquisition. During each of the 40 acquisitions of $100 \mathrm{~ms}, 45 \mathrm{~ms}$ are used to acquire one high-resolution image ( $\mathrm{rtx} 1), 30 \mathrm{~ms}$ for one pair of Doppler spectra (LDV) and the rest for the closed-loop AO. The trigger button of the rtx 1 simultaneously starts recording the images on the $\mathrm{rtx} 1$ and the Doppler acquisition, which is controlled by a dedicated LabVIEW software based on a version developed for a laser Doppler flowmeter. ${ }^{34}$

\subsubsection{Signal analysis}

The power spectrum is given by $\left\{s_{k}\right\}$, where $1 \leq k \leq N$. Considering the ideal case of a rectangular distribution of the power spectrum, the normalized function $r_{c}$ of partial summation:

$$
r_{c}(i)=\frac{\sum_{k=1}^{k=i} s_{k}}{\max \left(r_{c}\right)^{2}}
$$

will linearly increase up to the maximum power spectrum and then remain constant (Fig. 2 dashed lines). First, the horizontal line is found with a linear fit of the last $1 / 3$ of the $\left\{s_{k}\right\}$, for which only noise is expected. Then a second linear fit is done from $k=1$ to $k_{x}$, corresponding to a $10 \%$ change between the first linear fit and the $r_{c}$ function. The intersection of both fitted lines is assumed to be the maximum frequency shift of the spectrum.

\subsubsection{Image analysis}

The measurement of the vessel diameter close to the probing spot was implemented in MATLAB. Images are 16-bit gray scale with a resolution of $1392 \times 1040$ pixels, corresponding to roughly $1.6 \mu \mathrm{m}$ per pixel at the fundus. First, a threshold is applied at $80 \%$ of the maximal image intensity, and the probing beam spot is detected as the largest connected component. Then a $360 \times 360$ pixels square region of interest is set around the spot centroid, and a second-order polynomial is fitted to the intensity data to model the low-frequency variations due, among others, to the non-uniform illumination. The subsequent processing is applied on the difference between the original intensity values and the fitted model. The vessel orientation and the offset of its centerline with respect to the spot centroid are found by correlation with 24 precomputed synthetic vessel templates ( $15 \mathrm{deg}$ increment). Finally, with this rough vessel orientation, image profiles are extracted across the vessel centerline with an interprofile distance of two pixels. The distance in pixels between the vessel edges is determined for each profile. As last step, the vessel diameter is estimated as the mean value of the non-aberrant edge distances determined on each profile (see Fig. 3). The conversion from $n$ pixels to physical distance $d$ is based on the publication of Bennet ${ }^{35}$ and was provided by Imagine Eyes ${ }^{\mathscr{Q}}$ : 


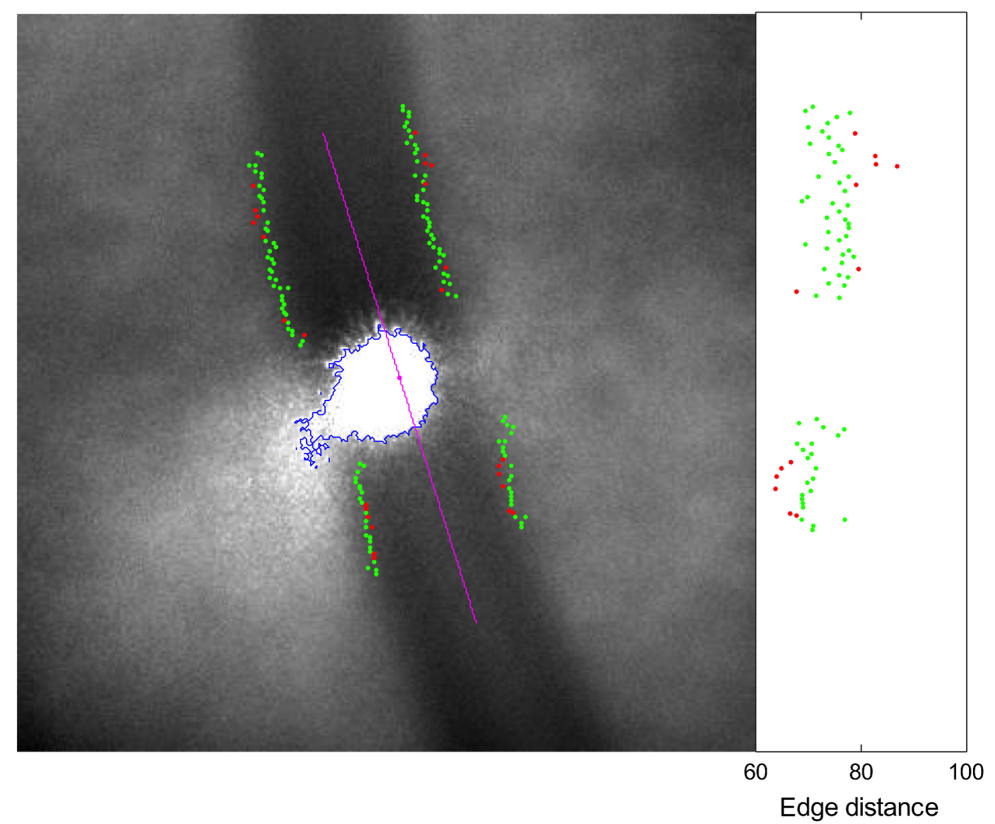

Fig. 3 Results of the image processing steps: the detected probing beam spot is the region bordered by the blue points. The spot centroid is in the center of a $360 \times 360$ pixels region of interest. The detected vessel orientation and position is represented by the straight line in magenta and the edges by the green and red points superimposed on the image. The distance between each pair of points is drawn in the graph on the right. Points drawn in red correspond to aberrant edge distances (values outside mean $\pm 3 \mathrm{sd}$ ). The mean distance between the green points is used to estimate the vessel diameter; 73.3 pixels in that case.

$$
d=n \frac{19.269(L-1.82)}{373.87} \mu \mathrm{m},
$$

where $L$ is the axial length in millimeters of the eye under examination.

\subsubsection{Calculation of mean flow rate}

Assuming a Poiseuille flow in a cylindrical cross section, the mean flow rate was calculated using the following equation:

$$
F=\pi\left(\frac{d}{2}\right)^{2} \cdot \frac{1}{2} v_{\max }
$$

where $d$ is the diameter of the vessel obtained from the image analysis and $v_{\max }$ is the maximum velocity of the RBCs in the vessel, which is derived from Eq. (1). The blood flow rate $F$ deviates by about $4 \%$ per $1 \mathrm{~mm}$ change in eye length.

\subsubsection{Measurement procedure}

The subject was seated in front of the rtx 1 and looked inside the rtx 1 objective. A high-resolution image of the retina was displayed by the rtx 1 to observe the selected vessel and the LDV probing beam. To observe retinal vessels close to the optic nerve head or outside the posterior pole, the range of movement of the internal rtx 1 fixation point was not large enough, and therefore, the left eye was fixating on an external point, positioned $1.5 \mathrm{~m}$ behind the $\mathrm{rtx} 1$.

Once the probing beam was moved on a first- or second-order retinal vessel in the temporal part of the fundus, the DOE was pushed into the optical path, converting the point into a line, which was then aligned with the retinal vessel by rotating the DP. Finally, the DOE was pulled out. 
The signals from the detectors were also connected to a loud speaker, so cardiac pulsed sound could be heard if the beam was focused on a retinal artery. The longitudinal beam position on the retinal vessel was optimized by varying the imaging focus plane until the spectra showed a clear cutoff (see Sec. 4) and the acoustic signal corresponded to a Doppler sound. The lateral position of the beam was adjusted with the external fixation point within about $20 \mu \mathrm{m}$.

\subsubsection{In Vitro measurements on latex microspheres flowing in a glass capillary}

We connected a glass capillary (drummond microcaps, inner diameter $200 \mu \mathrm{m}$ ) via a plastic tube to a syringe pump (Agilia SP, Fresenius Kabi, USA). Latex microspheres ( $1 \mu \mathrm{m}$ in diameter, Polysciences Europe GmbH, Eppelheim, Germany) suspended in water (180 $\mu \mathrm{L}$ of microsphere in $35 \mathrm{~mL}$ of water) were pushed through the capillary with the syringe. Further, a focusing lens ( $F=25.4 \mathrm{~mm}$, LB1761-B, Thorlabs) was mounted to focus the collimated probing beam on the capillary. This capillary-lens assembly was placed in front of the rtx1 objective. The vesselsimulating capillary was displayed on the rtx1 screen.

Linearity was asssed by comparing the Doppler measurement of the maximum velocity of microspheres with the precise flow set by the syringe pump.

\subsubsection{In Vivo measurements on venous junctions}

The study was conducted in accordance with the declaration of Helsinki for research involving human subjects and adhered to Good Clinical Practice guidelines. Written informed consent was obtained from the subjects after explaning the study. The study protocol was approved by the local Institutional Review Board (IRB \#6705). For this exploratory study, no optimal sample was calculated, but six healthy subjects with a very good fixation ability, between the age of 27 and 58 , participated in this study. A complete ocular examination, including slit lamp biomicroscopy, indirect funduscopy, fundus photography, and axial length measurement partial coherence interferometry (Zeiss IOL Master, Carl Zeiss Meditec Inc, Dublin, CA, USA), was conducted before the beginning of the study. Velocity measurements were conducted after dilatation of the right eye using a $1 \%$ topical tropicamide (Théa, Clermont-Ferrand, France). The rtx1 allows for an easy superposition of the pupil of the eye with that of the instrument.

To test the accuracy of the LDV instrument, blood flow in a retinal venous junction of each human volunteers was measured before the junction and in the two daughter vessels. The measurement before the junction was compared with the sum of the measurements of the daughter vessels to assert their equality. An exemple of a vein and its daughter veins is illustrated in Fig. 4.

\section{Results}

\subsection{In Vitro Measurements on Latex Microspheres Flowing in a Glass Capillary}

The Doppler measurements performed on the glass capillary were found to be between 0.92 and $13.3 \mathrm{~mm} / \mathrm{s}$ for the velocity and between 1.75 and $25.9 \mu \mathrm{L} / \mathrm{min}$ for the flow rate.

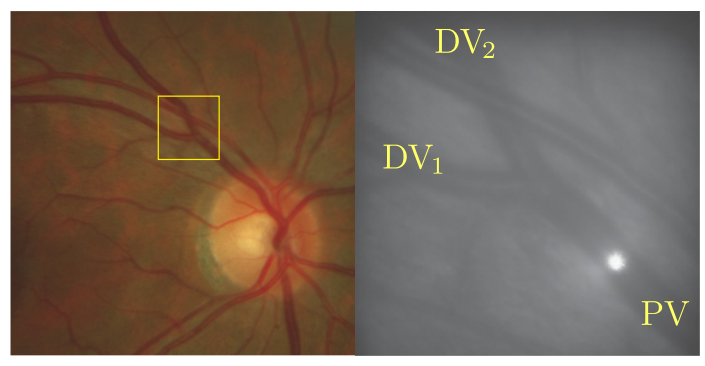

Fig. 4 Fundus image and corresponding $A O$ raw image obtained with the rtx 1 of a bifurcation measured with aoLDV. $P V$ is the principal vein that furcates into daugther veins $D V_{1}$ and $D V_{2}$. 


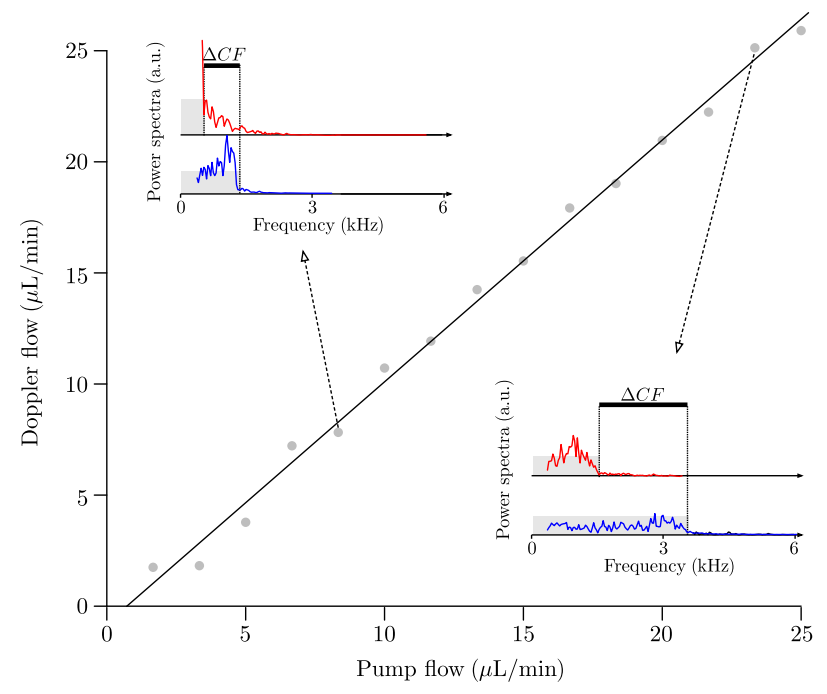

Fig. 5 Comparison between the difference in frequency shifts ( $\triangle \mathrm{CFs}$ ) obtained with the aoLDV on microspheres flowing in the capillary tube and the flow given by the syringe-pump (correlation coefficient $r^{2}=0.995$ ). On the power spectra, the peak before the cutoff is due to the light intensity being stronger in the center of the capillary than at the edges. Note that the total power spectrum (area under the curve) remained constant with the increase in velocity.

The measurements were highly correlated (slope equals 1.09, $r^{2}=0.995, p<0.0001$, see Fig. 5) with the flow imposed by the syringe pump.

\subsection{In Vivo Measurements on Venous Junctions}

Measured diameters of the retinal veins varied between 89 and $129 \mu \mathrm{m}$ (mean $104 \mu \mathrm{m}$ ) (Table 1). The velocity ranged from 2.3 to $21.1 \mathrm{~mm} / \mathrm{s}$ (mean $11.0 \mathrm{~mm} / \mathrm{s}$ ). The blood flow values in the daughter and parent vessels ranged from 0.9 to $13.2 \mu \mathrm{L} / \min$ (mean $5.7 \mu \mathrm{L} / \min$ ). The standard deviation of $\Delta F / F_{\mathrm{PV}}$ was $18.5 \%$.

\section{Discussion}

The development of this new prototype combining LDV and high-resolution AO imaging showed that (1) the LDV could be added using a complementary optical pathway; (2) optoelectronic development allowed for synchronization between both devices and therefore allowed for AO images and Doppler spectrum acquisitions every $0.1 \mathrm{~s}$; (3) experimental measurements using glass capillary and microspheres showed high correlations between measurements and imposed values; and (4) preliminary measurements in human retinal vessels showed results with acceptable errors.

Table 1 Measurements of RBF at retinal venous bifurcation in healthy subjects. $\Delta F$ is the difference between the flow in the parent vessel $\left(F_{\mathrm{PV}}\right)$ and the sum of the flow in the daughter vessels $\left(F_{\mathrm{DV} 1}\right.$ and $\left.F_{\mathrm{DV} 2}\right): \Delta F=F_{\mathrm{PV}}-\left(F_{\mathrm{DV} 1}+F_{\mathrm{DV} 2}\right)$. Analysis was done automatically. Flow units are $\mu \mathrm{L} / \mathrm{min}$.

\begin{tabular}{lcccccc}
\hline \hline Subject & $\mathrm{A}$ & $\mathrm{B}$ & $\mathrm{C}$ & $\mathrm{D}$ & $\mathrm{E}$ & $\mathrm{F}$ \\
\hline$F_{\mathrm{DV} 1}$ & 1.4 & 0.9 & 8.6 & 4 & 10.4 & 8.3 \\
$F_{\mathrm{DV} 2}$ & 3.4 & 1 & 6.8 & 3 & 1.7 & 3.5 \\
$F_{\mathrm{PV}}$ & 4.6 & 2.1 & 11.3 & 5.9 & 12.8 & 13.2 \\
$\Delta F / F_{\mathrm{PV}}(\%)$ & 4.8 & -8.3 & 36.1 & 19 & -6.3 & -10.7 \\
\hline \hline
\end{tabular}




\subsection{In Vitro Measurements on Latex Microspheres Flowing in a Glass Capillary}

To mimic a $130-\mu \mathrm{m}$ human retinal vessel, a $200-\mu \mathrm{m}$ glass capillary with a lens of $25.4 \mathrm{~mm}$ focal length was used. Capillaries in air with larger diameters would be preferable because they disturb the $\mathrm{AO}$ of the rtx1 less than smaller diameters. Our results show a clear linear relationship between the flow calculated with the Doppler measurements and the flow rate given by the syringe pump.

\subsection{In Vivo Measurements on Venous Junctions}

Our measurements of RBF were in the same range as those reported in previous studies of retinal vessels with similar diameters. Using a device based on the same fundamental principle, a bidirectional laser Doppler velocimeter, and monochromatic fundus photographs, Riva et al. ${ }^{16}$ measured retinal vessel diameters between 64 and $177 \mu \mathrm{m}$, corresponding to flow rate between 1 and $20 \mu \mathrm{L} / \mathrm{min}$, which is consistent with our measurements. Yoshida et al. ${ }^{36}$ used a canon laser blood flowmeter (CLBF100) and measured the blood flow rate to be between 3.2 and $14.4 \mu \mathrm{L} / \min$ (mean $8.3 \mu \mathrm{L} / \mathrm{min}$ ) in retinal veins with a diameter ranging from 98 to $166 \mu \mathrm{m}$ (mean, $138 \mu \mathrm{m}$ ) (see Table 2). In their study, Werkmeister et al. ${ }^{18}$ measured retinal veins between 84 and $172 \mu \mathrm{m}$ and found RBF between 1.9 and $18.7 \mu \mathrm{L} / \mathrm{min}$ with dual-beam bidirectional Doppler Fourier-domain optical coherence tomography.

\section{3 aoLDV Advantages and Feasibility}

aoLDV offers the possibility to perform very precise measurements of the inner diameter of vessels. Averaging 40 raw images, obtained from one measurement with the rtx1, each with a lateral pixel-resolution of $1.6 \mu \mathrm{m},{ }^{39}$ gives a better lateral pixel-resolution of $0.8 \mu \mathrm{m} .^{40}$

In this study, raw images were used to calculate the diameter since each image corresponds to a pair of Doppler spectra. In our study, 94 out of 480 raw images (19.6\%) were excluded from analysis, 31 (6.5\%) because they could not be analyzed (mainly due to blinks) and $63(13.1 \%)$ because the probing beam was not on the vessel. At the present time, no method to quantitatively assess perfusion is used in routine practice. This is due to the difficulties in performing RBF in vivo.

The evaluation of a new method of RBF measurement is difficult in the absence of a gold standard. An LDV system was implemented by canon (CLBF100), ${ }^{36}$ but only a few instruments

Table 2 Comparaison between our measurements and the results from different publications.

\begin{tabular}{lcccccccc}
\hline \hline Publ. & Techn. & Subj. Nb & Age (year) & Eyes Nb & Veins Nb & Dia $(\mu \mathrm{m})$ & Vel $(\mathrm{mm} / \mathrm{s})$ & Flow $(\mu \mathrm{L} / \mathrm{min})$ \\
\hline & aoLDV & 6 & $29(25-31)$ & 6 & 18 & $(89-129)$ & $0.9-13.3$ & $(121)$ \\
16 & LDV & 7 & $34(20-45)$ & 12 & 66 & $(64-177)$ & $(0.5-3.6)$ & $(120)$ \\
37 & LDV & 1 & $(21-43)$ & - & - & $156 \pm 20$ & $1.71-0.33$ & $12.5 \pm 3.5$ \\
38 & LDV & 12 & $27 \pm 5$ & - & - & $(132-176)$ & $(1.2-2.1)$ & $(7.818 .7)$ \\
15 & LDV & 64 & $31.8 \pm 6.5$ & - & - & $(90-185)$ & $(0.8-2.4)$ & $12.2 \pm 7^{\mathrm{a}}$ \\
& & & & & & & & $17.9 \pm 7.1^{\mathrm{b}}$ \\
36 & LBF & 6 & $(28-43)$ & 12 & 18 & $(98-166)$ & - & $(3-14)$ \\
18 & FD-OCT & 10 & $29(19-35)$ & 10 & 30 & $(84-172)$ & - & $(2-19)$ \\
20 & FD-OCT & 4 & $(20-30)$ & - & 73 & $(60-160)$ & - & $(1-16)$ \\
\hline \hline
\end{tabular}

Mean (min - max) or mean \pm standard deviation.

${ }^{\mathrm{a}}$ Temporal sup.

${ }^{\mathrm{b}}$ Temporal inf. 
were sold. Nevertheless, LDV has not reached routine clinical practice, in part because of alignement difficulties and because of the relative imprecision of diameter measurements. ${ }^{41}$ The spatial resolution is limited by the quality of the eye under observation.

OCT scanning is becoming standard in clinical optical imaging. ${ }^{42}$ OCT has a lateral resolution of around 12 to $20 \mu \mathrm{m}$ and an axial resolution between 2.3 and $7 \mu \mathrm{m}$. The price of such cameras is about five times higher than classical fundus cameras, but they offer many advantages of which the most interesting is its ability to build axial slices of the fundus. By adapting the detection, OCT allows for Doppler measurement. However, the speed of the scanning limits the range of measurable velocities. Our proposed system encompasses this shortcoming since we do not scan the retina.

\section{4 aoLDV Limitations}

We have encountered some limitations for the measurements of $v_{\max }$ on human subjects, including the need for a good ocular fixation for $4 \mathrm{~s}$ to ensure that the probing and detection beams, respectively, hit and come back from the center of the vessel (Fig. 6). Owing to slight eye movements, we may underestimate $v_{\max }$ by measuring velocities of RBCs close to the vessel wall (lower than $v_{\max }$ ) or by adding Doppler shifts associated with the surrounding tissue.

The AO live video of the fundus allowed us to verify the exact location of the laser beam on the vessel, but it did not provide the detection plane of the aoLDV, which could be different due to the $\mathrm{AO}$ adaptation.

Bidirectional LDV measurement accuracy strongly depends on the plane of focus (conjugate to the detection plane) of the probing beam as well as A and B beams (Fig. 6) and the position of the probing beam, which should be exactly on the vessel center. This causes a parallax problem, which occurs when the image plane of the LDV is not on the same image plane as the rtx 1 (Fig. 6). Obtaining a usefull pair of good spectra was a strong technical limitation. The constraint of the existing fundus camera rtx 1 prevents us from using the path of the AO for the LDV, leaving this path uncorrected for eye aberrations. To overcome this parallax problem, we manually change the refraction correction of the rtx 1 between -0.5 and 3 diopters to find the best correction to match the detection plane. These settings were time-consuming, $1 \mathrm{~h}$ for one measurement of a bifurcation.

The power spectrum from a glass capillary with a flowing fluid is typically a step function, in which the maximum of the frequency shift correponds to $v_{\max }$ (cutt-off frequency). The quality of the in vitro spectrum obtained with the moving light scattering microspheres was excellent, and the cutoff frequency was always well-defined. Another difficulty was calculating the precise cutoff frequency of the in vivo spectrum, which, most of the time, did not have a clear step shape. Thus $v_{\max }$ extraction was dependent on the algorithm to calculate the maximum of the frequency shift.
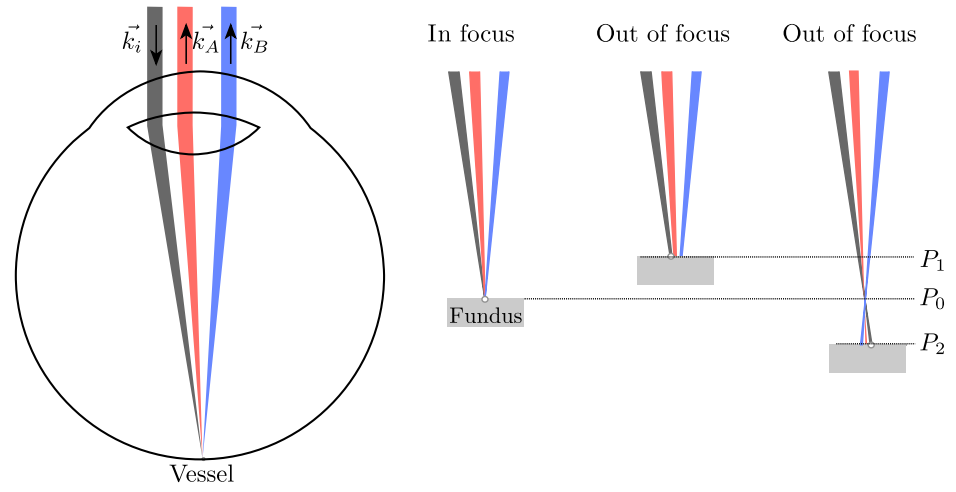

Fig. 6 Illustration of the parallax phenomenon, which explains that one or both scattered collected beams $\left(\vec{k}_{A}\right.$ and $\left.\vec{k}_{B}\right)$ can be out of focus while the laser beam $\left(\vec{k}_{i}\right)$ is focused on the vessel (circle on the fovea). $P_{0}$ is the image plane of the $\mathrm{rtx} 1$, and $P_{0}, P_{1}$, or $P_{2}$ are possible image planes of the LDV optical system. 


\subsection{Clinical and Technical Perspectives}

More studies with a larger series of subjects are needed to evaluate the reproducibility and to estimate the total RBF after measuring the main retinal vessels near the optic nerve disc. To allow for examination of both eyes with the rtx1, an external fixation target should be designed. A more robust image analysis with deep learning would reject fewer images. The development of software combining data from the Doppler spectra, the quality of AO images, and the exact location of the probing beam on the retinal vessel is in progress and will allow for real-time evaluation of measurements.

Assuming a correct focus, two illuminating beams $-\vec{k}_{A}$ and $-\vec{k}_{B}$ instead of collected scattered beams must hit at the fovea the same point as the probing beam $\vec{k}_{i}$ (Fig 6). This would solve the parallax issue.

\section{Conclusion}

In conclusion, this aoLDV prototype allows for simultaneous measurement of the inner retinal diameter and the velocity of the RBC within that vessel, which allows for real-time calculation of absolute blood flow rate. The main limitations of this prototype were due to optomechanical constraints, which prevents the use of the optical path of AO for the LDV. This produced occasional discrepancies between the focal planes of the AO and LDV optical paths (parallax effect), which suppressed Doppler signal in one or both channels. Improving the analysis method and solving the parallax issue will offer a high potential for examining RBF abnormalities in patients with ocular or systemic diseases. The results obtained in healthy subjects are promising.

\section{Disclosures}

Frederic Truffer, Martial Geiser, Helene Strese, and Christophe Chiquet are inventors of the patent FR3042963A1 and US10561314B2 (Dispositif de mesure de la vitesse du sang dans un vaisseau sanguin de l'oeil. Device for measuring the blood velocity in a blood vessel of the eye). This research was funded in part by Novartis. There are no other patents or products in development to declare. None of the authors have a competing interest even if some of the authors are inventors of the patent FR3042963A1 (Dispositif de mesure de la vitesse du sang dans un vaisseau sanguin de l'oeil. Device for measuring the blood velocity in a blood vessel of the eye).

\section{Acknowledgments}

Novartis Pharma S.A.S. (France) provided research funding for the preclinical part of this study. Association for research and teaching in Ophthalmology, Grenoble, France (ARFO AOLDV_2017) and University of Applied Sciences and Arts of Western Switzerland provided research and exchanges funding (No. 75678). Grenoble Alpes University Hospital, DRCI provided funding for human experiments (AOLDV 14.175). HES-SO gave partial salary to Frederic Truffer, Helene Strese, Gilbert Maître, and Serge Amoos. All other authors received no specific funding for this work. The funders had no role in study design, data collection and analysis, decision to publish, or preparation of the manuscript. There are not patents or products in development to declare. All authors thanks Charles Riva for many discussions and helpful remarks.

\section{References}

1. C. J. Pournaras et al., "Regulation of retinal blood flow in health and disease," Prog. Retinal Eye Res. 27(3), 284-330 (2008).

2. Z. Burgansky-Eliash et al., "Retinal blood flow velocity in patients with age-related macular degeneration," Curr. Eye Res. 39(3), 304-311 (2014). 
Truffer et al.: Absolute retinal blood flowmeter using a laser Doppler velocimeter combined...

3. R. Ehrlich et al., "Age-related ocular vascular changes," Graefes Arch. Clin. Exp. Ophthalmol. 247(5), 583-591 (2009).

4. B. Pemp and L. Schmetterer, "Ocular blood flow in diabetes and age-related macular degeneration," Can. J. Ophthalmol. 43(3), 295-301 (2008).

5. M. Sehi et al., "Retinal blood flow in glaucomatous eyes with single-hemifield damage," Ophthalmology 121(3), 750-758 (2014).

6. C. J. Pournaras and C. E. Riva, "Retinal blood flow evaluation," Ophthalmologica 229(2), 61-74 (2013).

7. J. E. Grunwald et al., "Total retinal volumetric blood flow rate in diabetic patients with poor glycemic control," Invest. Ophthalmol. Vis. Sci. 33(2), 356-363 (1992).

8. M. Ritt et al., "Impaired increase of retinal capillary blood flow to flicker light exposure in arterial hypertension," Hypertension 60(3), 871-876 (2012).

9. S. Gutfreund et al., "Retinal blood flow velocity in metabolic syndrome," Graefes Arch. Clin. Exp. Ophthalmol. 251(6), 1507-1513 (2013).

10. M. Lim et al., "Systemic associations of dynamic retinal vessel analysis: a review of current literature," Microcirculation 20(3), 257-268 (2013).

11. B. Pemp et al., "Effects of antioxidants (AREDS medication) on ocular blood flow and endothelial function in an endotoxin-induced model of oxidative stress in humans," Invest. Ophthalmol. Vis. Sci. 51(1), 2-6 (2010).

12. E. Rechtman et al., "An update on retinal circulation assessment technologies," Curr. Eye Res. 27(6), 329-343 (2003).

13. C. Riva, B. Ross, and G. B. Benedek, "Laser Doppler measurements of blood flow in capillary tubes and retinal arteries," Invest. Ophthalmol. 11(11), 936-944 (1972).

14. C. E. Riva et al., "Bidirectional LDV system for absolute measurement of blood speed in retinal vessels," Appl. Opt. 18(13), 2301-2306 (1979).

15. G. Garhöfer et al., "Retinal blood flow in healthy young subjects," Invest. Ophthalmol. Vis. Sci. 53(2), 698-703 (2012).

16. C. E. Riva et al., "Blood velocity and volumetric flow rate in human retinal vessels," Invest. Ophthalmol. Vis. Sci. 26(8), 1124-1132 (1985).

17. J. P. S. Garcia, P. T. Garcia, and R. B. Rosen, "Retinal blood flow in the normal human eye using the canon laser blood flowmeter," Ophthalmic Res. 34(5), 295-299 (2002).

18. R. M. Werkmeister et al., "Measurement of absolute blood flow velocity and blood flow in the human retina by dual-beam bidirectional Doppler Fourier-domain optical coherence tomography flow velocity values with bidirectional Doppler FD-OCT," Invest. Ophthalmol. Vis. Sci. 53(10), 6062-6071 (2012).

19. R. A. Leitgeb et al., "Doppler optical coherence tomography," Prog. Retinal Eye Res. 41(C), 26-43 (2014).

20. V. Doblhoff-Dier et al., "Measurement of the total retinal blood flow using dual beam Fourier-domain Doppler optical coherence tomography with orthogonal detection planes," Biomed. Opt. Express 5(2), 630-642 (2014).

21. C. L. Chen and R. K. Wang, "Optical coherence tomography based angiography," Biomed. Opt. Express 8(2), 1056-1082 (2017).

22. W. J. Choi et al., "Characterizing relationship between optical microangiography signals and capillary flow using microfluidic channels," Biomed. Opt. Express 7, 2709-2728 (2016).

23. L. Puyo et al., "In vivo laser Doppler holography of the human retina," Biomed. Opt. Express 9(9), 4113-4129 (2018).

24. D. D. Duncan and S. J. Kirkpatrick, "Can laser speckle flowmetry be made a quantitative tool?" J. Opt. Soc. Am. A 25(8), 2088-2094 (2008).

25. Y. Wang et al., "In vivo total retinal blood flow measurement by Fourier domain Doppler optical coherence tomography," J. Biomed. Opt. 12(4), 041215 (2007).

26. Y. Wang et al., "Retinal blood flow measurement by circumpapillary Fourier domain Doppler optical coherence tomography," J. Biomed. Opt. 13(6), 064003 (2008).

27. N. Patton et al., "Retinal image analysis: concepts, applications and potential," Prog. Retinal Eye Res. 25(1), 99-127 (2006).

28. F. Formaz, C. E. Riva, and M. Geiser, "Diffuse luminance flicker increases retinal vessel diameter in humans," Curr. Eye Res. 16(12), 1252-1257 (1997). 
29. E. Koch et al., "Morphometric analysis of small arteries in the human retina using adaptive optics imaging: relationship with blood pressure and focal vascular changes," J. Hypertension 32(4), 890-898 (2014).

30. C. Viard et al., "Imaging microscopic structures in pathological retinas using a floodillumination adaptive optics retinal camera," Proc. SPIE 7885, 788509 (2011).

31. B. Sahin et al., "Adaptive optics with pupil tracking for high resolution retinal imaging," Biomed. Opt. Express 3(2), 225-239 (2012).

32. E. Logean, L. Schmetterer, and C. E. Riva, "Velocity profile of red blood cells in human retinal vessels using confocal scanning laser Doppler velocimetry," Laser Phys. 13(1), 45-51 (2003).

33. H. L. Liou and N. A. Brennan, "Anatomically accurate, finite model eye for optical modeling," J. Opt. Soc. Am. A Opt. Image. Sci. Vis. 14(8), 1684-1695 (1997).

34. M. H. Geiser et al., "Schlieren laser Doppler flowmeter for the human optical nerve head with the flicker stimuli," J. Biomed. Opt. 18(12), 127001 (2013).

35. A. G. Bennett, A. R. Rudnicka, and D. F. Edgar, "Improvements on Littmann's method of determining the size of retinal features by fundus photography," Graefes Arch. Clin. Exp. Ophthalmol. 232(6), 361-367 (1994).

36. A. Yoshida et al., "Reproducibility and clinical application of a newly developed stabilized retinal laser Doppler instrument," Am. J. Ophthalmol. 135(3), 356-361 (2003).

37. J. E. Grunwald, "Effect of two weeks of timolol maleate treatment on the normal retinal circulation," Invest. Ophthalmol. Vis. Sci. 32(1), 39-45 (1991).

38. S. J. Pakola and J. E. Grunwald, "Effects of oxygen and carbon dioxide on human retinal circulation," Invest. Ophthalmol. Vis. Sci. 34(10), 2866-2870 (1993).

39. D. Rosenbaum et al., "Imaging of retinal arteries with adaptative optics, feasibility and reproducibility," Ann. Cardiol. Angeiol. (Paris) 62(3), 184-188 (2013).

40. N. Lermé et al., "Segmentation of retinal arteries in adaptive optics images," in 22nd Int. Conf. Pattern Recognit., IEEE, pp. 574-579 (2014).

41. D. Baleanu et al., "Wall-to-lumen ratio of retinal arterioles and arteriole-to-venule ratio of retinal vessels in patients with cerebrovascular damage," Invest. Ophthalmol. Vis. Sci. 50, 4351-4359 (2009).

42. D. F. Kiernan, W. F. Mieler, and S. M. Hariprasad, "Spectral-domain optical coherence tomography: a comparison of modern high-resolution retinal imaging systems," Am. $J$. Ophthalmol. 149(1), 18-31.e2 (2010).

Frederic Truffer has a background in electrical and optical systems engineering and has worked for more than 13 years in the development of ophthalmology instruments. His focus is on laser Doppler flowmeters for human and small animals and he is strongly involved in projects with an absolute laser Doppler velocimeter.

Martial Geiser, physicist, has more than 25 years of experience in lens design and optical instrumentation with numerous publications. His research is focused on optical instrumentation such as laser Doppler flowmetry or velocimetry, which has led to not only the recent development of compact flowmeters but also fluorescence sensing for microfluidic systems and multispectral imaging of the skin.

Marc-Antoine Chappelet, fellow in ophthalmology, was strongly involved in the human clinical use of AO-LDV, in the clinical research team of Grenoble-Alpes University Hospital. His experience in retinal retina allowed him to successfully schedule the first measurements in humans and obtained a master of science.

Helene Strese received a Bachelor of Engineering in optics from the University of Applied Sciences of Jena in Germany in 2013 and a Master of Science from the University of Applied Sciences of Western Switzerland in 2017. She specialized in lens design.

Gilbert Maître received his MSc degree in electrical engineering from Swiss Federal Institute of Technology Zurich and his PhD from the University of Neuchâtel in 1994. Since 2005, he has been a research associate at the University of Applied Sciences of Western Switzerland, Sion. 
His main research interests are signal and image processing, as well as pattern recognition and machine learning.

Serge Amoos is a senior scientist at the HES-SO. He has been working for several decades in the field of mechanical engineering, particularly in the development of industrial or laboratory equipment, as well as in the design of optical and medical devices. His skills range from preparation of specifications to rapid prototyping, manufacturing, and industrialization.

Florent Aptel is a specialist of glaucoma at the University Hospital of Grenoble-Alpes and Department of Ophthalmology. He is highly involved in many projects in glaucoma from diagnosis, physiopathology, intraocular pressure measurements, treatment, and ocular blood flow.

Christophe Chiquet is the head of the Department of Ophthalmology and Visual Sciences at the University Hospital of Grenoble-Alpes. His main interests include medical and surgical retina and uveitis. He developed a multimodal approach for the study of ocular blood flow, using laser Doppler velocimetry, adaptive optics, OCTA imaging, and computerized analysis of retinal vessels measurements. 